\title{
Prevalence of Intestinal Parasites Among Preschool Children and Maternal KAP on Prevention and Control in Senbete and Bete Towns, North Shoa, Ethiopia
}

\author{
Moges Lewetegn ${ }^{1}$, Meron Getachew ${ }^{2}$, Tadesse Kebede ${ }^{3}$, Gemechu Tadesse $^{4}$, Tsegahun Asfaw ${ }^{1,}$ * \\ ${ }^{1}$ Department of Medical Laboratory Science, Debre Berhan University, Debre Berhan, Ethiopia \\ ${ }^{2}$ Bloomberg Philanthropies Initiative for Global Road Safety Surveillance Coordinator, Addis Ababa, Ethiopia \\ ${ }^{3}$ Department of Microbiology, Immunology, and Parasitology, Addis Ababa University, Addis Ababa, Ethiopia \\ ${ }^{4}$ Department of Parasitology, Ethiopian Public Health Institute, Addis Ababa, Ethiopia
}

Email address:

mogeslt@gmail.com (M. Lewetegn), merget2004@yahoo.com(M. Getachew),tkas2003@gmail.com (T. Kebede), gemechut_2006@yahoo.com(G. Tadesse), tsegahun.asfaw12@gmail.com (T.Asfaw)

${ }^{*}$ Corresponding author

\section{To cite this article:}

Moges Lewetegn, Meron Getachew, Tadesse Kebede, Gemechu Tadesse, Tsegahun Asfaw. Prevalence of Intestinal Parasites Among Preschool Children and Maternal KAP on Prevention and Control in Senbete and Bete Towns, North Shoa, Ethiopia. International Journal of Biomedical Materials Research. Vol. 7, No. 1, 2019, pp. 1-7. doi: 10.11648/j.ijbmr.20190701.11

Received: January 10, 2019; Accepted: February 11, 2019; Published: February 27, 2019

\begin{abstract}
In developing countries, intestinal parasites like protozoa and helminths are highly prevalent in preschool children. There is also poor understanding of the mother's knowledge, attitude, and practices towards parasitic infections. Therefore, this study is designed to assess the prevalence of intestinal parasite and maternal knowledge, attitude and practice on the prevention and control of intestinal parasites. Cross-sectional study was conducted on preschool children in Senbete and Bete towns. Stool specimens were collected and examined for intestinal parasites by using Kato-Katz and formol-ether concentration technique. Mother's knowledge, attitude, and practice data were collected using a per-tested structured questionnaire. Data was analysed using SPSS-20 and P values less than 0.05 was considered as statistically significant value. Among 214 preschool children, the overall prevalence of intestinal parasite was $52.3 \%$. The predominant parasites was Hymenolepis nana (23.8 \%), followed by Giardia lamblia (19.6\%). Among 214 interviewed mothers 129 $(60.3 \%)$ had knowledge on prevention and control of intestinal parasites. And also $120(56.1 \%)$ of the respondent had positive attitude on the prevention and control of intestinal parasites. Moreover, 95(44.4\%) of the mothers used toilet or container to dispose their children's faeces and 186(86.9\%) mothers gave drug for their child. High prevalence of intestinal parasite was found. Maternal education level, open field defecation and playing with soil were significantly associated with intestinal parasitic infections. Therefore, health education program to improve maternal knowledge, attitude and practice should be implemented.
\end{abstract}

Keywords: Preschool Children, Intestinal Parasite, Maternal Knowledge, Attitude and Practice

\section{Background}

Intestinal parasites are major public health problem in several developing countries. According to World Health Organization (WHO), over 1.5 billion people are infected with one or more intestinal parasites. Moreover, 700 million people infected with hookworm and 807 million people infected with ascariasis [1]. Intestinal parasites are more predominant in the developing countries mostly in subSaharan Africa [2]. In Ethiopia there is high burden of intestinal parasites. The overall national prevalence of any helminths infection was $29.8 \%$ with variable degree of prevalence among regions [3].

Intestinal parasite infections are common among 
preschool children with different causes such as playing with soil, sucking fingers and defecation in open field. Maternal awareness for the prevention and control of intestinal parasite has its own impact on the prevalence. To reduce the impact of intestinal parasites, increasing access to safe water, sanitation and health education are necessary [2]. WHO also recommends periodic preventive chemotherapy like albendazole or mebendazole as a public health intervention [1]. Globally in 2013, more than 266 million preschool-aged and 609 million school-aged children were estimated in need of preventive chemotherapy for soil transmitted helminths in 106 countries. In Africa more than 13.8 million preschool aged children in need of treatment were treated [1].

In Ethiopia the main strategies are mass drug administration, case detection and transmission control. However, information on the prevalence and distributions of intestinal parasites is incomplete and not updated periodically. Even there is no enough study throughout the country. Therefore this study is designed to assess the prevalence of intestinal parasite and maternal Knowledge, attitude and practice on the prevention and control of intestinal parasites.

\section{Methods}

\subsection{Study Design and Period}

A community based cross- sectional study was conducted in July 2018.

\subsection{Study Area}

Senbete and Bete towns are found in Jile timuga woreda, North Showa zone, Amhara region, Ethiopia. The annual average range of temperature in Senbete and Bete towns is $24-30^{\circ} \mathrm{C}$ and annual rainfall of approximately 500-700 $\mathrm{mm}$. The area has an altitudinal of 1000 to $1450 \mathrm{~m}$. The total population in Senbete and Bete town is 7,047 and 2,105 respectively according to Jile timuga woreda health office.

\subsection{Eligibility Criteria}

All mothers with children (1-5 years) living in Senbete and Bete towns at least for 1 year and willing to participate in the study was included while mothers having children taking standard intestinal parasite treatment for previous month and children who has seriously diseases were excluded.

\subsection{Data Collection}

Socio-demographic data and mother's knowledge, attitude and practice were collected with a structured questionnaire by trained health workers.

Sample collection, handling and transportation

Orientation was given for mother to collect about $2 \mathrm{~g}$ fresh stool sample from their own preschool child using clean, dry and well labelled specimen cup. Then samples were transport to Bete town health centre laboratory. In health centre laboratory a portion of the sample was processed by Katokatz method using a template delivering a plug of $41.7 \mathrm{mg}$ of stool as described by Nyantekyi [4]. The remaining sample was preserved in test-tube containing $10 \%$ formalin. All preserved samples and Kato- katz slides were transported to Ethiopian Public Health Institute (EPHI) parasitology laboratory and examined by using formal-ether concentration technique.

\subsection{Quality Control}

For each steps Standard Operational Procedure (SOP) was followed. Microscopic reading was done by senior laboratory technologists. Data quality was assured by prior training of data collectors about the objective of the study and data collection procedure. In addition quality control was performed with daily checking.

\subsection{Statistical Analyses of Data}

The data was analysed by using SPSS-version 20. Frequency and cross tabulation were used to summarize descriptive statistics of the data. Finally the association between variables was identified by OR, 95\% CI and Pvalue.

\subsection{Ethical Consideration}

Ethical approval was obtained from Research and Review Committee of Addis Ababa University. Permission was obtained from Jile timuga woreda. Informed consent was obtained from each child's mother. Children with intestinal parasitic infection were treated with appropriate drug and dose for each parasites obtained.

\subsection{Operational Definitions}

Attitude: assessment of mothers opinion, thought about intestinal parasite prevention and control.

1. Positive attitude: mothers who responded below the mean $(<9.2)$.

2. Negative attitude: mothers who respond above the mean $(>9.2)$.

Our questioner prepared using Likert scales for attitude questions. Calculation was based on maximum score scaling to include all responses. The maximum response is 5 and the minimum response 20 . The mean response of respondent was 9.2. If all questions reposes are strongly agree, the score will be 5 and strongly disagree the score will be 20 (range is between 5 and 20). The order is 1 for strongly agree, 2 for agree, 3 for disagree and 4 for strongly disagree on the questionnaire.

Knowledge: assessment of what mothers understanding about intestinal parasites prevention and control. The following definitions were used to score the level of understanding. The scoring method was adapted from Abera H, and Tebeje B. 2009 [5].

1. Knowledgeable: scoring of $80 \% \quad-100 \%$ from knowledge measuring questions about IP prevention 
and control. If the mother answered $>7$ knowledge measuring questions.

2. Fairly knowledgeable: scoring from $50 \%-79 \%$ of knowledge measuring questions. If the mother answered 5-7 knowledge measuring questions.

3. Non-knowledgeable: scoring $<50 \%$ of knowledge measuring questions. If mother answered $<5$ knowledge measuring questions.

Practice: assessment of mother's exercises on the prevention and control of intestinal parasite.

\section{Result}

\subsection{Maternal and Children Socio-Demographic Status}

A Total of 214 mothers whose children are able to produce stool sample were included in this study. Mean age of mothers was 27.5 (SD 5.5) years. Almost all 199 (93\%) of study participants were married. More than three forth $(79 \%)$ of the mothers did not attained formal education. The majority of study participants (74.3\%) have between 4-6 family members. And also a total of 214 children were enrolled of which 104 $(48.6 \%)$ were male and $110(51.4 \%)$ were female. The mean ages of the children were 3.4 (SD 1.1) years (Table 1).

Table 1. Maternal and children socio-demographic status Senbete and Bete towns, 2018.

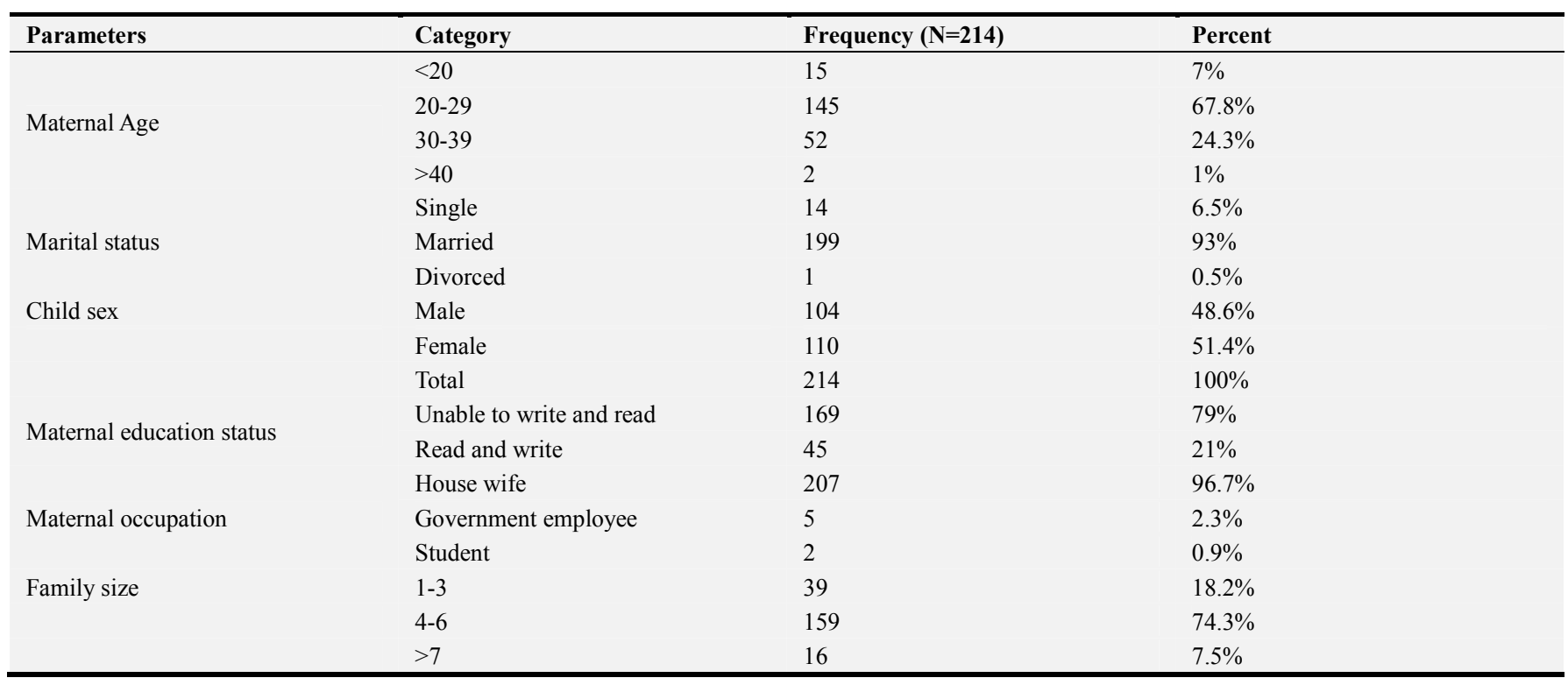

\subsection{The Total Prevalence of Intestinal Parasites in Senbete and Bete Towns}

Among 214 preschool children, 112 (52.3\%) were infected with one or more intestinal parasites. The major protozoan parasites identified were Giardia lamblia (19.2\%) and Entamoeba histolytica/E.dispar (8.4\%). The total prevalence of Ascaris lumbricoides, Enterobius vermicularis, Hymenolepis nana and Schistosoma mansoni was 5.1\%, $1.9 \%, 21.5 \%$ and $4.2 \%$ respectively as determined by Kato- katz and formol-ether concentration methods. (Figure 1).

\subsubsection{Prevalence of Intestinal Parasites Detected by Kato-Katz Result and Intensity of Infections}

Using kato-katz method three helminths were detected. The most frequently identified parasites was H. nana (14.5\%) followed by $S$. mansoni (4.2\%) and A.lumbricoides $1.4 \%$. Among children where $9 \mathrm{~S}$. mansoni detected, 7 of them had light infection while two had moderate infections (Table $2 \&$ 3).

Table 2. Prevalence of intestinal parasites by Kato-katz techniques in Senbete and Bete towns 2018.

\begin{tabular}{lll}
\hline Parasite name & Frequency & Present \\
\hline H. nana & 31 & $14.5 \%$ \\
Lumbricoides & 4 & $1.4 \%$ \\
S. mansoni & 9 & $4.2 \%$ \\
No ova/parasite seen & 170 & $79.4 \%$ \\
Total & 214 & $100 \%$ \\
\hline
\end{tabular}

Table 3. Intensity of helminths infections among under-five children in Senbete and Bete towns 2018

\begin{tabular}{llll}
\hline Organisms & Light & Moderate & Infection status threshold* \\
\hline A.lumbricoides $(\mathrm{n}=4)$ & 1 & 0 & Light (1- 4999EPG), Moderate (5000- 49999) \\
S.mansoni $(n=9)$ & 7 & 2 & Light (1-99EPG), Moderate (100-399EPG0 \\
\hline
\end{tabular}

* EPG: Eggs per gram of Stool, Schistosomiasis and STH, (light, moderate and heavy) based on WHO threshold (WHO, 2002). 


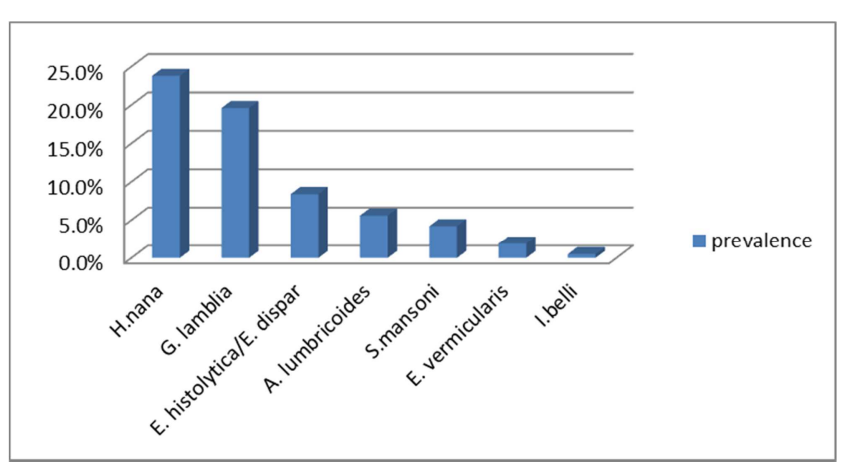

Figure 1. Prevalence of intestinal parasites in Senbete and Bete towns 2018.

\subsubsection{Prevalence of Intestinal Parasites by Formol-Ether Concentration Technique}

Among 214 preschool children, 108(50.5\%) were positive for intestinal parasites by formol-ether concentration technique where, $90(42.1 \%)$ of the children were infected with one intestinal parasites and $18(8.4 \%)$ of the children were infected with two intestinal parasites. The total prevalence of helminths was $66(30.8 \%)$ while protozoa was $60(28.0 \%)$. Among helminths, the most frequently identified was $H$. nana (14.5\%), while among protozoa was $E$. histolytica/E. dispar 14 (6.5) (Table 4).

Table 4. Prevalence of intestinal parasites by formol-ether concentration techniques in Senbete and Bete towns 2018.

\begin{tabular}{ll}
\hline Types of parasites & Frequency (\%) \\
\hline Protozoa & \\
E. histolytica/E. dispar & $14(6.5)$ \\
G. lamblia & $32(15)$ \\
Helminths & \\
H.nana & $29(13.6)$ \\
A. lumbricoides & $8(3.7)$ \\
E. vermicularis & $4(1.9)$ \\
S.mansoni & $3(1.4)$ \\
Mixed infection & \\
H.nana and G. lamblia & $9(4.2)$ \\
E. histolytica/E. dispar and H.nana & $3(1.4)$ \\
H.nana and A. lumbricoides & $3(1.4$ \\
I. belli and H.nana & $1(0.5)$ \\
S. mansoni and H.nana & $1(0.5)$ \\
E. histolytica/E. dispar and S.mansoni & $1(0.5)$ \\
Total Number of helminths & $66(30.8)$ \\
Total number of Protozoa & $60(28.1)$ \\
No o/p & $106(49.5)$ \\
Total & $214(100)$ \\
\hline
\end{tabular}

\subsection{Knowledge on the Prevention and Control of Intestinal Parasites}

Among 214 interviewed mothers 129 (60.3\%) had knowledge on prevention and control of intestinal parasites. While, 85(39.7\%) hadn't knowledge. Knowledge score result showed that $41(19 \%)$ mothers are knowledgeable, $88(40 \%)$ are non-knowledgeable and 88 (41\%) fairly-knowledgeable (Table 5 \& Figure 2).

Table 5. Mothers' response on selected questions about intestinal parasites prevention and control in Senbete and Bete towns 2015.

\begin{tabular}{lll}
\hline Knowledge Variables & Yes n (\%) & No n (\%) \\
\hline $\begin{array}{l}\text { Do you know what intestinal parasites are? } \\
\text { Eating contaminated food }\end{array}$ & $170(79.4)$ & $44(20.6)$ \\
Transmission & $151(70.6)$ & $63(29.4)$ \\
Walking bare foot & $49(22.9)$ & $165(77.1)$ \\
Lack of hygiene & $132(61.7)$ & $82(38.3)$ \\
Sign and symptom & & \\
Abdominal pain & $130(60.7)$ & $84(39.3)$ \\
Diarrhea & $74(34.6)$ & $140(65.4)$ \\
Wight loss & $32(15)$ & $182(85)$ \\
Prevention and control & & \\
Washing hand before eating Prevent IP & $152(71)$ & $62(29)$ \\
Taking de worming drug & $115(53.7)$ & $99(46.3)$ \\
Using clean toilet & $116(54.2)$ & $98(45.8)$ \\
\hline
\end{tabular}

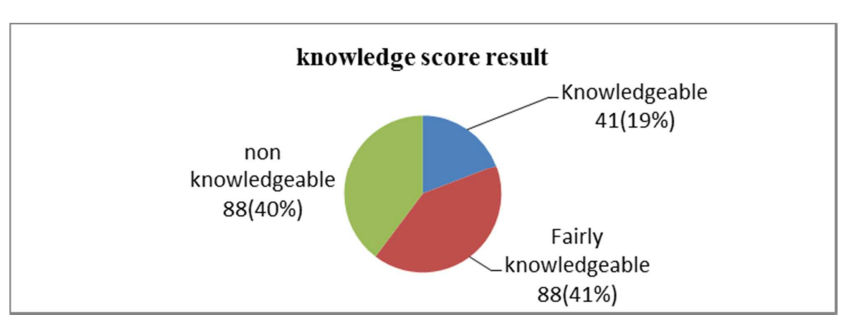

Figure 2. Knowledge score on the IP prevention and control among mothers.

\subsection{Attitude of Mothers Towards Prevention and Control of Intestinal Parasites}

Among 214 mothers $120(56.1 \%)$ had positive attitude while, 94(43.9\%) had negative attitude towards the prevention and control of intestinal parasites. Almost half of the mothers $104(48.6 \%)$ were strongly agreed that, lack of hygiene is the cause of infection with intestinal parasites. Ninety five $(44.4 \%)$ of the mother have strongly agree with attitude towards using soap when washing hand is preventive for intestinal parasite infection (Table 6).

Table 6. Attitude of mothers towards prevention and control of intestinal parasites in Senbete and Bete towns 2015.

\begin{tabular}{|c|c|c|c|c|}
\hline Variable & Strongly Agree n (\%) & Agree n (\%) & Strongly Disagree n (\%) & Disagree n (\%) \\
\hline Lack of hygiene is cause for IPs & $104(48.6)$ & $75(35)$ & $4(1.9)$ & $31(14.5)$ \\
\hline We can prevent and treat IPs disease? & $93(43.5)$ & $85(39.7)$ & $6(2.8)$ & $30(14)$ \\
\hline Health education can reduce IPs prevalence? & $102(47.7)$ & $78(36.4)$ & $5(2.3)$ & $29(13.6)$ \\
\hline $\begin{array}{l}\text { If IPs untreated, it can transmit to other family member and } \\
\text { cause growth retardation }\end{array}$ & $99(46.3)$ & $71(33.2)$ & $7(3.3)$ & $37(17.3)$ \\
\hline Uses soap when washing hands is preventive for IPs & $95(44.4)$ & $89(41.6)$ & $6(2.8)$ & $24(11.2)$ \\
\hline
\end{tabular}




\subsection{Mothers' Practice on the Prevention and Control of Intestinal Parasites}

Half of the mothers $(52.3 \%)$ had children infected with intestinal parasites at least one time in their life. 95(44.4\%) of the mothers used toilet or container to dispose their children's faeces. Moreover, 186(86.9\%) mothers gave drug for their child to prevent intestinal parasite (Table 7).

Table 7. Mothers' practice on the prevention and control of intestinal parasites Senbete and Bete towns 2015.

\begin{tabular}{lll}
\hline Practice & Yes n (\%) & No n (\%) \\
\hline Child who had any stool examination previously & $117(54.7)$ & $97(45.3)$ \\
Use of toilet or container for their child defecation & $95(44.4)$ & $119(55.6)$ \\
Wash fruit before consuming it & $158(73.8)$ & $56(26.2)$ \\
Cut nail when it grow & $155(72.4)$ & $59(27.6)$ \\
Wash children hand after defecation & $141(65.9)$ & $73(34.1)$ \\
Using chimerically treated, boiled or taps water & $161(75.2)$ & $53(24.8)$ \\
Using drugs for prevention of intestinal parasite & $186(86.9)$ & $28(13.1)$ \\
\hline
\end{tabular}

\subsection{Factors Associated with Total Prevalence of Intestinal Parasites}

Only mothers educational status $(\mathrm{P}=0.01)$ was associated with intestinal parasitic infections. Mothers who can read and write were less likely to have a child infected with parasitic infections compared to mothers who are unable read and write (OR-0.25 95\% CI-0.08-0.72) (Table 8).

Table 8. Factors associated with total prevalence of intestinal parasites.

\begin{tabular}{|c|c|c|c|c|c|c|}
\hline \multirow{2}{*}{ Variable } & \multirow{2}{*}{ Character } & \multicolumn{2}{|c|}{ IP prevalence } & \multirow[t]{2}{*}{$\operatorname{COR}(95 \% \mathrm{CI}) *$} & \multirow[t]{2}{*}{ AOR(95\%CI)* } & \multirow[t]{2}{*}{ Sig } \\
\hline & & YES (\%) & NO (\%) & & & \\
\hline \multirow[t]{2}{*}{ Child sex } & Male & $48(46)$ & $56(53)$ & $0.62(0.36-1.06)$ & $1.6(0.92-2.86)$ & 0.09 \\
\hline & Female & $64(58)$ & $46(41)$ & 1.000 & 1.000 & \\
\hline \multirow{2}{*}{ Maternal education } & Unable to write and read & $80(47)$ & $89(52)$ & 1.000 & 1.000 & \\
\hline & Read and write & $32(71)$ & $13(29)$ & $0.23(0.45-0.59)$ & $2.4(0.18-0.56)$ & 0.002 \\
\hline \multirow{2}{*}{ Using toilet or container. } & Yes & $56(58)$ & $39(41)$ & $0.62(0.35-1.06)$ & $0.7(0.4-1.32)$ & 0.3 \\
\hline & No & $56(47)$ & $63(52)$ & 1.000 & 1.000 & \\
\hline Using chemically treated, boiled water. & No & $33(62)$ & $20(37)$ & 1.000 & 1.000 & \\
\hline
\end{tabular}

* $\mathrm{COR}=$ Crude odds ratio and $\mathrm{AOR}=$ Adjusted odds ratio.

\subsection{Factors Associated with Intestinal Helminths Infections}

In bivariate analysis educational status, having knowledge on meaning of intestinal parasite, using toilet or container for their child defecation, mothers who wash fruit before consuming, mothers who cut their child nail and using chemically treated, boiled or tap water were associated with intestinal helminths having $(\mathrm{P}<0.2)$. In multivariate logistic regression, maternal education level and using toilet or container for their child defecation were significantly associated with intestinal helminths (Table 9).

Table 9. Factors associated with intestinal helminths infections.

\begin{tabular}{|c|c|c|c|c|c|c|}
\hline \multirow{2}{*}{ Variable } & \multirow{2}{*}{ Character } & \multicolumn{2}{|c|}{ Helminths } & \multirow{2}{*}{$\operatorname{COR}(95 \% \mathrm{CI})$} & \multirow{2}{*}{$\operatorname{AOR}(95 \% C I)$} & \multirow{2}{*}{ Sig } \\
\hline & & Yes $(\%)$ & No $(\%)$ & & & \\
\hline \multirow{2}{*}{ Educational status } & Unable to read & $50(30)$ & $119(70)$ & 1.000 & 1.000 & \\
\hline & Read and write & $20(44)$ & $25(56)$ & $0.28(0.12-0.67)$ & $0.45(0.24-0.92)$ & 0.003 \\
\hline \multirow{2}{*}{ Having knowledge what IP means } & Yes & $55(32)$ & $117(68)$ & $1.18(0.58-2.4)$ & $1.2(0.58-2.82)$ & 0.54 \\
\hline & No & $15(35)$ & $17(64)$ & 1.000 & 1.0000 & \\
\hline \multirow{2}{*}{ Use toilet or container } & Yes & $39(41)$ & $56(59)$ & 1.000 & 1.000 & 0.041 \\
\hline & No & $31(26)$ & $88(73)$ & $1.9(1.11-3.5)$ & $1.85(1.02-3.34)$ & \\
\hline \multirow{2}{*}{ Wash fruit } & Yes & $56(35)$ & $102(64)$ & $0.61(0.3-1.2)$ & $0.75(0.25-2.23)$ & 0.609 \\
\hline & No & $14(25)$ & $42(75)$ & 1.000 & 1.0000 & \\
\hline \multirow{2}{*}{ Cutting their child nail } & Yes & $55(35)$ & $100(64)$ & $0.62(0.3-1.2)$ & $0.72(0.26-2.02)$ & 0.53 \\
\hline & No & $15(25)$ & $44(74)$ & 1.000 & 1.000 & 1.000 \\
\hline \multirow{2}{*}{ Using chimerically treated, boiled water } & Yes & $48(30)$ & $113(70)$ & $0.6(0.31-1.14)$ & $1.21(0.75-2.82)$ & 0.27 \\
\hline & No & $22(41)$ & $31(58)$ & 1.000 & 1.000 & \\
\hline
\end{tabular}

\subsection{Factors Associated with Intestinal Protozoan Infections}

Mothers who washed fruit before eating, child who have habit of playing with soil and cutting nail when it grow were associated with intestinal protozoa in bivariate analysis ( $p$-value $<0.2$ ). In multivariate logistic regression, children who had 
habit of playing with soil had two times higher odds of being infected with intestinal protozoa compare those without such habits (OR-2.01 95\% CI 1.04-3.8) (Table 10).

Table 10. Determinants of protozoan intestinal parasitic infections.

\begin{tabular}{|c|c|c|c|c|c|c|}
\hline \multirow{2}{*}{ Variable } & \multirow{2}{*}{ Character } & \multicolumn{2}{|l|}{ Protozoa } & \multirow{2}{*}{ COR(95\%CI) } & \multirow{2}{*}{ AOR(95\%CI) } & \multirow{2}{*}{ p-value } \\
\hline & & Yes $(\%)$ & No $(\%)$ & & & \\
\hline \multirow{2}{*}{ Washing fruit before eating } & Yes & $38(24)$ & $120(76)$ & $2.13(1.17-4.04)$ & $2.44(0.89-6.73)$ & 0.08 \\
\hline & No & $22(40)$ & $34(60)$ & 1.000 & 1.000 & \\
\hline \multirow{2}{*}{ Habit of playing with soil } & Yes & $38(26)$ & $106(74)$ & $1.6(0.86-2.9)$ & $2.01(1.04-3.83)$ & 0.04 \\
\hline & No & $24(34)$ & $46(66)$ & 1.000 & 1.000 & \\
\hline \multirow{2}{*}{ Cutting child nail regularly } & Yes & $40(25)$ & $115(74)$ & $1.7(0.90-3.23)$ & $1.06(0.40-3.15)$ & 0.91 \\
\hline & No & $20(34)$ & $39(66)$ & 1.000 & 1.000 & \\
\hline
\end{tabular}

\section{Discussion}

The findings of the present study showed that overall prevalence of intestinal parasitic infection among preschool children was $52.3 \%$ where Hymenolepis nana was the most prevalent helminths and Giardia lamblia was the most prevalent protozoan parasite. This finding is relatively higher than study done in Gamo area, south Ethiopia 29.4\% [6], Arbaminch town, Southern Ethiopia 27.9\% [7] and Kenya $25.6 \%$ (8). However, our report is lower than study done in Shesha Kekele, Wondo Genet, in Southern Ethiopia 85.1\% [4].

This study showed maternal education level, use of open field for defecation of their child and playing with soil was significantly associated with intestinal parasite. Variations in prevalence rates of intestinal parasites from different Ethiopian communities could be related to several factors including the educational level of the study population, personal and environmental hygiene and probably social habits such as use of toilet for children. In addition, some ecological factors such as temperature, relative humidity, rainfall could be responsible for observed differences in prevalence between communities.

Among 214 preschool children, 90 (42.1\%) of the children were infected with one intestinal parasites and 18 (8.4\%) of the children were infected with two intestinal parasites. Other study done in Shesha Kebele, Wondo Genet, in Southern Ethiopia also showed $34.5 \%, 33.3 \%$ and $23.2 \%$ had single, double and multiple parasitic infections, respectively [4]. The prevalence of single infections among preschool children was higher in highland and lowland dwellers in Gamo area, South Ethiopia $83.9 \%$. This difference might be due to small sample size [6]. However the present finding is in agreement with a study done in Senegal [9].

This study also revealed that among protozoan parasites, Giardia lamblia (19.2\%) was frequently observed followed by Entamoeba histolytica/E. dispar (8.4\%). Similarly other studies done in Arbaminch reported those two parasites $4.2 \%$ and $12.9 \%$, respectively [7] and also $10.6 \%$ and $11.4 \%$, respectively from the study in Gamo area [6]. However, the prevalence of Giardia lamblia is lower than study done in Mexican rural school children [11].

In this study, predominant helminthic intestinal parasites were Hymenolepis nana (21.4\%) and Ascaris lumbricoides (5.1\%). A study done in Gondar, Northwest Ethiopia reported
13.8\% Hymenolepis nana and 5.9\% Ascaris lumbricoides [10]. The same study done in Mexico and Egypt also showed, $H$. nana was predominant [11-12]. The observed differences might be from differences in sample size, study population and the methods used for diagnosis. Additional factors might be socio-demographic factors, climate and geographic difference.

In this study $73.4 \%$ of mothers took training on prevention and control of intestinal parasites. However, $60.3 \%$ mothers had knowledge on prevention and control of intestinal parasites. Our finding is comparable to the previous study done in Shesha Kekele, Wondo Genet, Southern Ethiopia [4]. However, in contrast to a previous study conducted in rural Malaysia where, the present study revealed higher knowledge response from the study participant [13]. This difference could be due to the study population, and the previous study focused only on soil-transmitted helminths.

In this study half of the mothers responded, their child was infected by intestinal parasite at least ones in his/her life time. However $44 \%$ of the mothers responded that they use toilet or a container to dispose their children's faeces and $86.9 \%$ mothers gave drug for their child to prevent intestinal parasite. Using toilet is preventive for intestinal parasites and deworming program by the government also contribute for the response of mothers.

In this study there is no significant association between intestinal parasitic infections and socio-demographic status of participating mothers or children. However, maternal education level is an important predicting factor for intestinal parasitic infections in children. The other study done in Mexican rural areas also indicated that less educated mothers had higher risk of intestinal parasites [11].

The current study also showed the association of open defecation and increased risk for helminths infections. Those families who practiced open defecation were two times more vulnerable for intestinal helminths (OR 2.01 95\% CI 1.023.34). This is supported by study done in Mexican rural areas [11]. According to this study properly functioning and cleaned toilets reduced helminths infections. Children who have habits of playing with soil had increased risk to be infected by protozoan parasites.

\section{Conclusion and Recommendations}

According to this study intestinal parasitic infections are a 
common health problem among preschool children. Maternal educational level, use of toilet or container for child defecation and habit of playing with soil were closely associated with the prevalence of intestinal parasitic infections. While the former two protects the children from infection, the latter predispose them. Therefore, long term control measures including health education and mass treatment should be given to reduce intestinal parasitic infections among preschool children.

\section{Conflict of Interests}

The authors declare that they have no conflict of interests.

\section{Funding}

This work was funded from Addis Ababa University core budget.

\section{Authors' Contributions}

ML-performed the laboratory activities. ML, MG, TK and GT- analysed the data. TA-wrote the manuscript. All authors read and approved the final manuscript.

\section{Acknowledgements}

We would like to thank Addis Ababa University for giving this opportunity and its fund. We also thank staffs and head of Bete health centre for their help during data collection process.

\section{References}

[1] World Health Organization (WHO) (2015). Weekly epidemiological record. Soil-transmitted helminthiases: number of children treated in 2013. 10 (90), 89-96.

[2] World Health Organization (WHO) (2002). Prevention and control of Schistosomiasis and soil transmitted helminthiasis. Report of WHO expert committee. Geneva.

[3] Federal Democratic Republic of Ethiopia/Mistry of Health (FDRE/MOH). National Master Plan for Neglected Tropical Diseases (NTDs) (2013-2015). Addis Ababa, Ethiopia.

[4] Nyantekyi L, Legesse M, Belay M, Tadesse K, Manaye K, Macias C, Erko B (2010). Intestinal parasitic infections among under-five children and maternal awareness about the infections in Shesha Kekele, Wondo Genet, Southern Ethiopia. Ethiop. J. Health Dev. 24 (3):185-190.

[5] Abera H and Tebeje B (2009). Knowledge, attitudes, and practices towards emergency contraception among female Jimma University students, Jimma, Southwest Ethiopia. Ethio. Journal of Reproductive Health. 1 (3), 37-43.

[6] Wegayehu T, Tsalla T, Seifu1B and Teklu T (2013). Prevalence of intestinal parasitic infections among highland and lowland dwellers in Gamo area, South Ethiopia. BMC Public Health. 13:151.

[7] Haftu D, Deyessa N, Agedew E (2014). Prevalence and determinant factors of intestinal parasites among school children in Arba Minch town, Southern Ethiopia; Am.J. of Health Research. 2 (5): 247-254.

[8] Mbae K, Nokes D, Mulinge E, Nyambura J, Waruru A and Kariuki S (2013). Intestinal parasitic infections in children presenting with diarrhoea in outpatient and inpatient settings in an informal settlement of Nairobi, Kenya. BMC Infectious Diseases. 13:243.

[9] Tine R, Faye B, Ndour C, Sylla K, Sow D, Ndiaye M, Ndiaye J, Magnussen P, Alifrangis M, Bygbjerg I et al. (2013) Parasitic Infections among Children under Five Years in Senegal: Prevalence and Effect on Anaemia and Nutritional Status. ISRN Parasitology http://dx.doi.org/10.5402/2013/272701.

[10] Gelaw A, Anagaw B, Nigussie B, Silesh B, Yirga A, Alem M, Endris M, Gelaw B, (2013). Prevalence of intestinal parasitic infections and risk factors among schoolchildren at the University of Gondar Community School, Northwest Ethiopia: a cross-sectional study. BMC Public Health. 13:304 http://www.biomedcentral.com/1471-2458/13/304.

[11] Quihui L, Valencia M, WT Crompton D, Phillips S, Hagan P, Morales G, Díaz-Camacho S (2006). Role of the employment status and education of mothers in the prevalence of intestinal parasitic infections in Mexican rural schoolchildren. BMC Public Health. 6:225 http://www.biomedcentral.com/1471$2458 / 6 / 225$.

[12] Curtale F, Pezzotti P, Sharbini A, Al Maadat H, Ingrosso P, Saad Y, Babille M (1998). Knowledge, perceptions and behaviour of mothers toward intestinal helminths in Upper Egypt: implications for control. Oxford University Press, 13 (4): 423-432.

[13] Nasr N, M Al-Mekhlafi H, Ahmed A, Roslan M, Bulgiba A (2013). Towards an effective control programme of soiltransmitted helminth infections among Orang Asli in rural Malaysia. Part 2: Knowledge, attitude, and practices. Parasites \& Vectors, 6:28. 